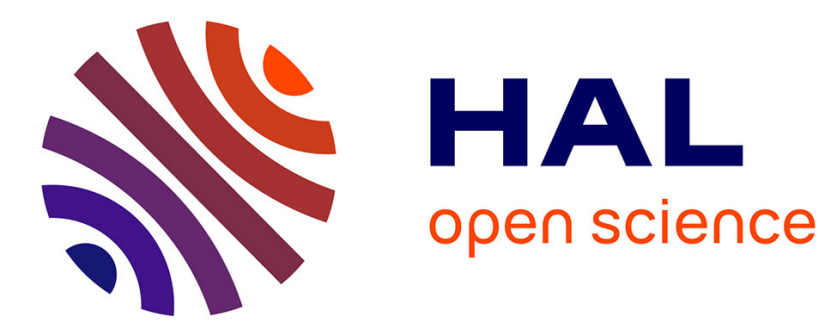

\title{
The dynamics of a developing CW supercontinuum: analytical predictions and experiments
}

\author{
J.M. Dudley, G. Genty, F. Dias, B. Kibler, N. Akhmediev
}

\section{To cite this version:}

J.M. Dudley, G. Genty, F. Dias, B. Kibler, N. Akhmediev. The dynamics of a developing CW supercontinuum: analytical predictions and experiments. Conference on Optital Fiber Communication (OFC)/Collocated National Fiber Optic Engineers (NFOEC), Jun 2010, San Fransisco, United States. 10.1364/OFC.2010.OTuJ7 . hal-00589620

\section{HAL Id: hal-00589620 https://hal.science/hal-00589620}

Submitted on 14 Apr 2021

HAL is a multi-disciplinary open access archive for the deposit and dissemination of scientific research documents, whether they are published or not. The documents may come from teaching and research institutions in France or abroad, or from public or private research centers.
L'archive ouverte pluridisciplinaire HAL, est destinée au dépôt et à la diffusion de documents scientifiques de niveau recherche, publiés ou non, émanant des établissements d'enseignement et de recherche français ou étrangers, des laboratoires publics ou privés. 


\title{
The dynamics of a developing CW supercontinuum: analytical predictions and experiments
}

\author{
John M. Dudley ${ }^{1}$, Goëry Genty ${ }^{2}$, Frederic Dias ${ }^{3}$, Bertrand Kibler $^{4}$, and Nail Akhmediev ${ }^{2}$ \\ ${ }^{1}$ Université de Franche-Comté, Institut FEMTO-ST, 25030 Besançon, France \\ ${ }^{2}$ Tampere University of Technology, Optics Laboratory, FI-33101 Tampere, Finland \\ ${ }^{3}$ Centre de Mathématique et de Leurs Applications (CMLA), ENS Cachan, France \\ ${ }^{4}$ CNRS/Université de Bourgogne, Institut Carnot de Bourgogne21078 Dijon, France \\ ${ }^{5}$ Australian National University, Institute of Advanced Studies, Canberra ACT 0200, Australia
}

\begin{abstract}
We show that the development of the supercontinuum spectrum in the quasi-CW regime can be interpreted analytically in terms of Akhmediev Breathers. Theory and experiment are in excellent agreement.

(C)2010 Optical Society of America

OCIS codes: (060.5530) Pulse propagation and temporal solitons; (060.5295) Photonic crystal fibers; (320.6629)

Supercontinuum generation
\end{abstract}

\section{Introduction}

Despite intensive research over three decades, there are surprisingly-few analytic solutions describing nonlinear pulse evolution in optical fibers. Certainly there are exceptions such as the well-known soliton and self-similar solutions [1,2], but complex processes such as supercontinuum generation have generally been considered to require numerical approaches [3]. In this paper, however, we report on the use of the "Akhmediev Breather" formalism to describe the development of the supercontinuum spectrum when seeded from spontaneous noise.

\section{Akhmediev Breather dynamics and the onset of supercontinuum generation}

The Akhmediev Breather (AB) is an exact analytic solution describing the evolution with distance of a modulated continuous wave with distance in an optical fiber [4]. In fact, the AB solution describes the dynamics of induced modulation instability (MI), and it is surprising that, although these results are nearly 25 years old, they have largely been overlooked in studies of MI and its applications. The AB evolution of a CW wave with small initial modulation yields a train of ultrashort pulses that undergoes an initial phase of temporal compression followed by subsequent temporal broadening. At the point of maximum temporal compression, the individual subpulses have maximum amplitude and minimum temporal width given by:

$$
A(z=0, T)=\sqrt{P_{0}} \frac{(1-4 a)+\sqrt{2 a} \cos \left(\omega_{\bmod } T\right)}{\sqrt{2 a} \cos \left(\omega_{\bmod } T\right)-1} .
$$

Here $2 a=\left[1-\left(\omega_{\text {mod }} / \omega_{\mathrm{c}}\right)^{2}\right]$ with $\omega_{\mathrm{c}}{ }^{2}=4 \gamma P_{0} /\left|\beta_{2}\right|$ with $\omega_{\text {mod }}$ and $\mathrm{P}_{0}$ the modulation frequency and peak power of the initial wave, and $\gamma$ and $\beta_{2}<0$ the fiber nonlinearity and group velocity dispersion. The corresponding spectrum consists of discrete frequency sideband modes with separation $\omega_{\text {mod }}$, and intensities that decrease following a geometric progression [4]. For the particular case of the maximally-compressed solution of Eq. (1), the spectral mode intensities are: $S_{0}=P_{0}\left(2^{1 / 2}-1\right)^{2}$ for the pump component, and $S_{\mathrm{n}}=2 P_{0}\left(2^{1 / 2}-1\right)^{2|\mathrm{n}|}$ (where $n= \pm 1, \pm 2$, $\pm 3, \ldots$ ) for the sidebands. Thus the pump and sideband intensities follow the relative progression $\left\{I_{0}, I_{1}, I_{2}, I_{3}\right.$, $\left.I_{4} \ldots\right\}=\{1,2,0.3431,0.0589,0.0101 \ldots\}$ so that there is a $3 \mathrm{~dB}$ increase from the pump $(\mathrm{n}=0)$ to the first sideband $(n= \pm 1)$ and then a constant decrease of $20 \log _{10}\left(2^{1 / 2}-1\right)=-7.66 \mathrm{~dB}$ between subsequent sidebands. This geometric progression describing the decrease in sideband amplitudes yields a characteristic triangular shape in the wings of the spectrum when plotted semi-logarithmically.

To discuss how this result can be used to interpret the onset dynamics of quasi-CW supercontinuum generation, we begin by showing experimental results: Fig. 1(a) shows spectral measurements at the output of $3.9 \mathrm{~m}$ of a highly nonlinear photonic crystal fiber with zero-dispersion wavelength at $780 \mathrm{~nm}$ for various peak powers using $1 \mathrm{~ns}$ pulses at $1064 \mathrm{~nm}$. Experiment (top) is compared with generalised nonlinear Schrödinger equation (GNLSE) simulations (bottom). The agreement between experiment and simulation is very good and illustrate how the spectral characteristics vary significantly with power. Firstly, at the lowest power of $26 \mathrm{~W}$, the spectrum consists of small number of distinct sidebands separated by the frequency of peak MI gain. As the power increases to $43 \mathrm{~W}$, we enter an "extended MI" regime where we can still resolve sidebands close to the pump, but we also see the development of continuous low amplitude wings that appear triangular when plotted with a semi-logarithmic y-axis. 
The spectral width in this regime approaches $60 \mathrm{~nm}$ at the $-40 \mathrm{~dB}$ level, and we note the first sign of a very low amplitude Raman peak around $1120 \mathrm{~nm}$. Finally at $98 \mathrm{~W}$, we see a significant increase in bandwidth to $400 \mathrm{~nm}$ at the $-40 \mathrm{~dB}$ level, and the spectrum assumes characteristics of a "fully-developed" supercontinuum [3].

(a) $26 \mathrm{~W}$

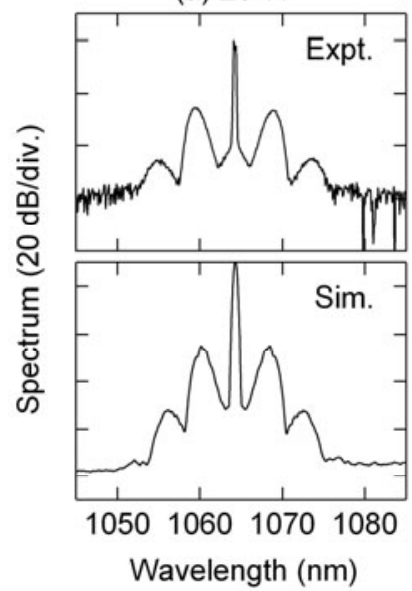

(b) $43 \mathrm{~W}$

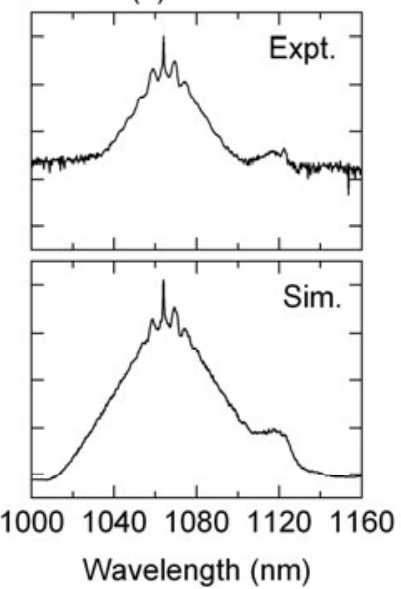

(c) $98 \mathrm{~W}$

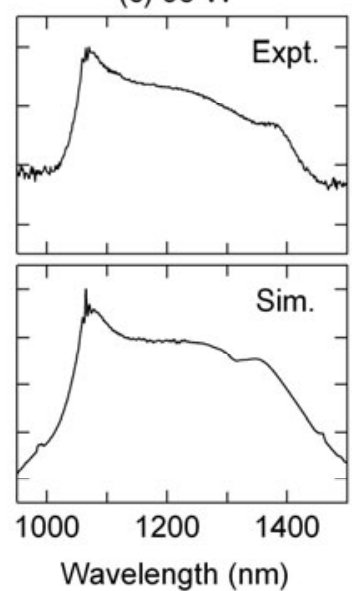

Fig. 1. Experimental (top) and simulation (bottom) results for $1 \mathrm{~ns}$ pulses at $1064 \mathrm{~nm}$ injected into highly nonlinear PCF at peak powers as shown. Simulation results are averaged and convolved with a resolution function matching the bandwidth of the spectrum analyzer used.

We next focus on the characteristics of the extended MI spectrum at $43 \mathrm{~W}$ peak power. This is a regime where we are in a "NLSE dynamics" regime, before the onset of higher-order effects. To examine how the AB theory can be applied to interpret the properties of the broadened spectrum seen at $43 \mathrm{~W}$, Fig. 2 presents simulation results showing in more detail how the (a) spectral and (b) temporal properties develop with distance.

(a) Spectral evolution

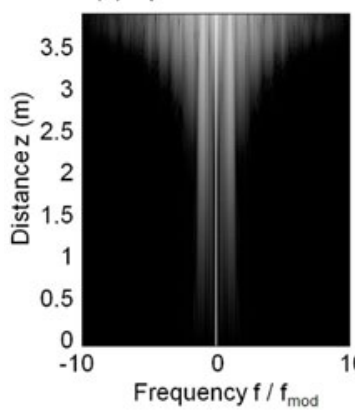

(b) Temporal evolution and expanded view of temporal profile
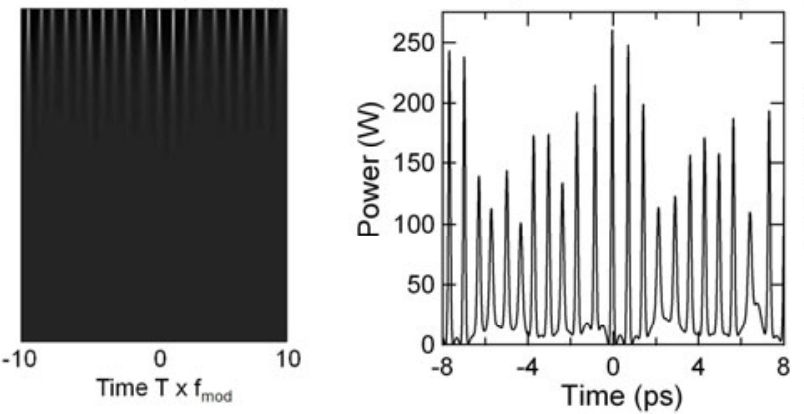

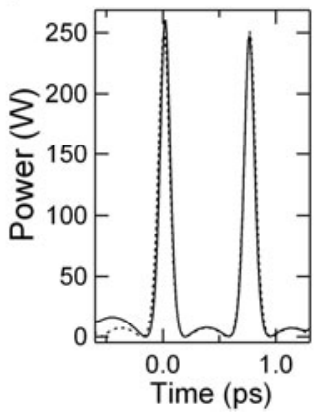

Fig. 2. Single shot (a) spectral and (b) temporal evolution. The shaded region in the extracted temporal trace is shown in detail in the rightmost figure, comparing simulations (solid line) with the AB solution at a modulation frequency corresponding to peak MI gain (dashed line).

The simulations use fiber parameters corresponding to experiment, and assume a $43 \mathrm{~W}$ peak power CW field with one photon per mode noise. The grayscale plots show results of a single numerical realization illustrating the field evolution over $3.9 \mathrm{~m}$. The spectral bandwidth first reaches its maximum extension at a distance of $3.5 \mathrm{~m}$ where we extract the corresponding temporal profiles. The extracted temporal profile shows a high contrast pulse train, but the broadband noise that seeds the MI results in significant variation in the structure of the individual temporal peaks across the extracted profile. Nonetheless, the highest amplitude peaks in the temporal profile are fitted very well by the maximally-compressed $\mathrm{AB}$ sub-pulses calculated from Eq. (1) using $\omega_{\text {mod }}$ corresponding to the peak of the MI gain $\left(f_{\text {mod }}=1.32 \mathrm{THz}\right.$ for our parameters. $)$ This agreement is shown explicitly in the expanded view of the shaded section in Fig. 2(b). In fact, analysis of the highest-amplitude peaks at other regions of the profile (not shown) reveals similarly good agreement with the calculated $\mathrm{AB}$ solution, and we have confirmed that simulations using different noise seeds reveal the same characteristics. 
The results in Fig. 2 suggest that the extended MI spectrum contains a dominant contribution from spontaneously-generated $\mathrm{AB}$ sub-pulses generated at the peak of the MI gain. In fact, this is also confirmed using experimental spectral measurements. Specifically, we compare the form of the measured spectrum with the analytic geometric progression predicted for the maximally-compressed $\mathrm{AB}$ as shown in Fig. 3. The figure compares numerical and analytic results with the experimental results of Fig. 1 for $P_{0}=43 \mathrm{~W}$. The numerical results show averaged multi-shot simulations using the full GNLSE with a 1 ns pulse input field (blue, short dashes) and using only the NLSE with a CW input field (red, long dashes). It is clear that both GNLSE and NLSE simulations produce similar results in agreement with experiment, with the GNLSE of course yielding better agreement in predicting the onset of a small Raman peak on the long wavelength edge around $1120 \mathrm{~nm}$.

The most significant result, however, is the agreement between experiment and the calculated amplitudes of the discrete frequency modes associated with the analytic form of the maximally-compressed AB. Because spontaneous MI is seeded by broadband noise, the spectral structure is not discrete, but rather consists of a continuous span of frequencies. Nonetheless, as with the time domain characteristics, we expect the spectral characteristics to contain a dominant component from the maximally-compressed AB solution calculated at peak MI gain. Specifically, normalising to the $n= \pm 1$ sideband amplitudes seen in experiment, the decay of spectral intensity with frequency in experiment and simulations is reproduced very well by the analytic geometric progression of the maximally-compressed AB breather.

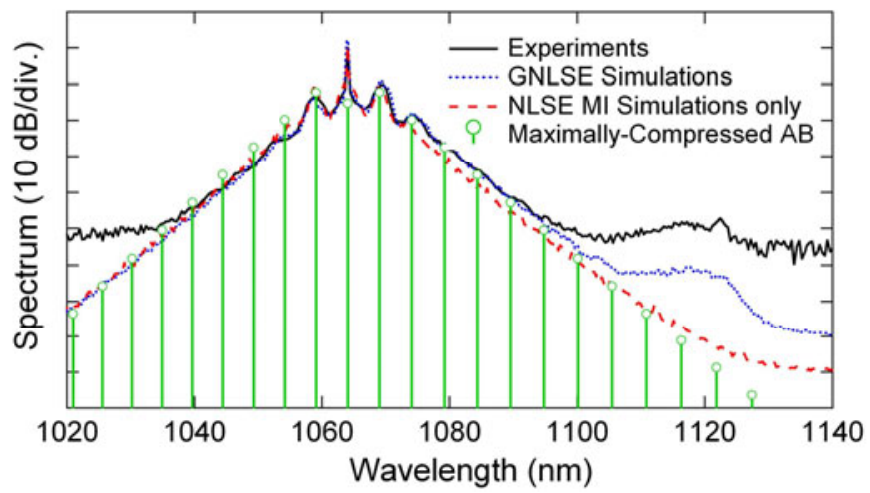

Fig. 3 Comparison between experiments (solid black line), numerical simulations using the full GNLSE (blue dashed line), numerical simulations using the NLSE only (red dashed line), and the calculated spectrum of the maximallycompressed $\mathrm{AB}$ (green lines from zero).

\section{Conclusions}

These studies of the temporal and spectral properties of spontaneous MI have shown that the characteristics of both the modulated temporal profile and the associated spectrum can be explained in terms of the development of high amplitude Akhmediev Breather sub-pulses. The analytic form of the AB spectrum has been shown to be in very good quantitative agreement with the wings of the spontaneous MI spectrum observed in experiments studying the onset of quasi-CW supercontinuum generation. These results represent an important step in applying the analytic formalism of breather evolution to the interpretation of supercontinuum generation. Moreover, in the case of induced MI from dual frequency fields, the $\mathrm{AB}$ theory would be expected to also be applied to fields such as optimization of pulse train and frequency comb generation from multiwave mixing processes [5].

\section{References}

[1] G. P. Agrawal, Nonlinear Fiber Optics. 4th Edition. Academic Press, Boston (2007).

[2] J. M. Dudley, C. Finot, D. J. Richardson, G, Millot, "Self Similarity in Ultrafast Nonlinear Optics", Nat.s Physics 3 597-603 (2007).

[3] J. M. Dudley, G. Genty, and S. Coen, "Supercontinuum Generation in Photonic Crystal Fiber”, Rev. Mod. Phys. 78, 1135-1184 (2006).

[4] N. Akhmediev and V. I. Korneev, "Modulation instability and periodic solutions of the nonlinear Schrodinger equation,” Theor. Math. Phys. 69, 1089-1093 (1986)

[5] B. Barviau, C. Finot, J. Fatome, G. Millot, "Generation from continuous waves of frequency combs with large overall bandwidth and tunable central wavelength,” Electron. Lett. 43, 886-887 (2007). 